\title{
The Demographic Dimensions of Fertility in Finland 1984
}

\author{
WOLFGANG LUTZ
}

Research Scholar

International Institute for Applied Systems Analysis

Laxenburg, Austria

\section{Events in the multi-dimensional demographic space}

The event of a human birth may be perceived and registered in relation to a wide range of criteria and background variables, demographic and non-demographic ones. Traditionally in demographic analysis the mother's age has been regarded as the basic underlying covariate of human fertility, but marital duration, length of birth interval, birth order, and several socioeconomic and other variables are often studied in their relationship to reproduction.

Once the event of a birth is unambiguously defined and it is clear to which universe it belongs, for demographers time becomes by far the most important dimension under which this event is registered and studied. In demography time usually has two distinct aspects: individual or personal time such as age and marital duration, and historical time which is measured across all individuals.

We may define individual or personal time as a clock that is started at the birth of a human being and goes on in counting the (intersubjectively measurable) hours until the person dies. At other demographically relevant events, i.e. marriage, birth of a child, divorce or death of spouse, etc., additional clocks start to run and count the time in the new status.

Independent from the individual clocks running, historical time is measured across all individuals of all age groups, marriage durations, or open birth intervals. Historical time is the standard for the analysis of trends, evolutions, or periodical fluctuations on an aggregate level. It is needless to say that historical time is also counted in objectively measurable time units of equal length. The fact that individual time (years of age or duration) and historical time (years of time) are measured by using the same unit is one of the deeper reasons for the analytical strength of demographic methodology. The homomorphism of these two time concepts allows demographers to construct synthetic cohorts and make period analyses comparable to cohort analyses.

All demographic processes over the life cycle of an individual depend on time. They always depend on both individual and historical time. In this study we are concerned with the events of births, and we will study births with respect to the individual times of the parents in certain year (1984) of historical time. Although a woman and a man are needed to create a new individual, demographic analysis so far has almost exclusively focused at the mother's characteristics and left out the father's altogether. The question of male fertility that is almost never raised in conventional demography but often comes up in interdisciplinary discussions. There are several good reasons to focus on the mother instead of the father. First, it is the woman that actually carries out the birth and the father cannot be identified in all cases (see 
the old Roman principle: pater semper incertus). Second, the age span during which individuals can have children is more clearly limited for women than for men which is convenient for statistical analyses; and finally demographers hardly have a chance to study male fertility because the appropriate data is lacking. All these considerations, however, do not fully justify the complete neglect of men in fertility analysis. In this study we will make an attempt to measure and analyze male fertility as well.

At the time of the birth several individual clocks are running for men and women. Generally three such individual times or durations have been considered to have some relevance for fertility: age; duration of marriage in case of marital fertility; and time since the last birth in case of parity one and above. We will consider these three demographic dimensions separately below and also give quantitative estimates of their relative impact on fertility. Since the present study will use period data, i.e. refer to one interval of historical time, the period mode of temporal aggregation (see Ryder, 1982) is the appropriate way to arrange the empirical period measured in Finland in 1984.

\section{Empirical period rates for Finland 1984}

The analysis in this short article is based on a cross-classification of the complete Finnish population on January 1, 1984 by sex, age, marital status, duration in marital status, parity, and duration in parity. ${ }^{1}$ For each of the cells the number of vital events during the year 1984 is given. Aside from the technical problems of treating such a large data set and some questions of definition such as in the case of adopted children and multiple births, etc., this is an ideal data set for studying the demographic dimensions of fertility under a period perspective.

\section{Age and marriage duration}

Figures 1 to 3 present three-dimensional perspectives of the empirical transition rates calculated from the data set described above. The figures are given in the form of contour maps that give two demographic dimensions on the axes while the intensity is represented by the shading of the specific area. The darker the shading the higher the birth rates. The labels on the axes (e.g. age and marital duration in Figure 1) refer to the individual times as they were measured at the beginning of the year 1984. For this reason we have to be aware of the fact that the actual exact age at a birth during 1984 of a woman that was of 20 years old (referring to her last birthday) on January 1, 1984 may range from 20 to just under age 22 (if she turned 20 on January 2, 1983 and had her birth on December 31, 1984). Hence a label of 20 might, on the average, refer to women with exact ages around 21 . The same is true with respect to marital duration and duration since last birth.

Figure 1 plots the distribution of marital births with respect to the two individual time dimensions age and marital duration. We have a priori knowledge about the effect of both dimensions: with respect to the mother's age we know that the reproductive period is bounded by menarche and menopause and that even uncontrolled fertility is expected to decline with age because of decreasing fecundability. In most societies procreation is closely linked to marriage and in contraceptive societies fertility is also limited in dependence of the number of children already born, so that

The author wishes to thank the Finnish Statistical Office and especially Mauri Nieminen for making these data available. This research was in part supported by a grant from the Austrian Science Foundation. 
F i g u r e 1. Shaded contour map of fertility rates specific for age and marital duration for married Finnish women in 1984.
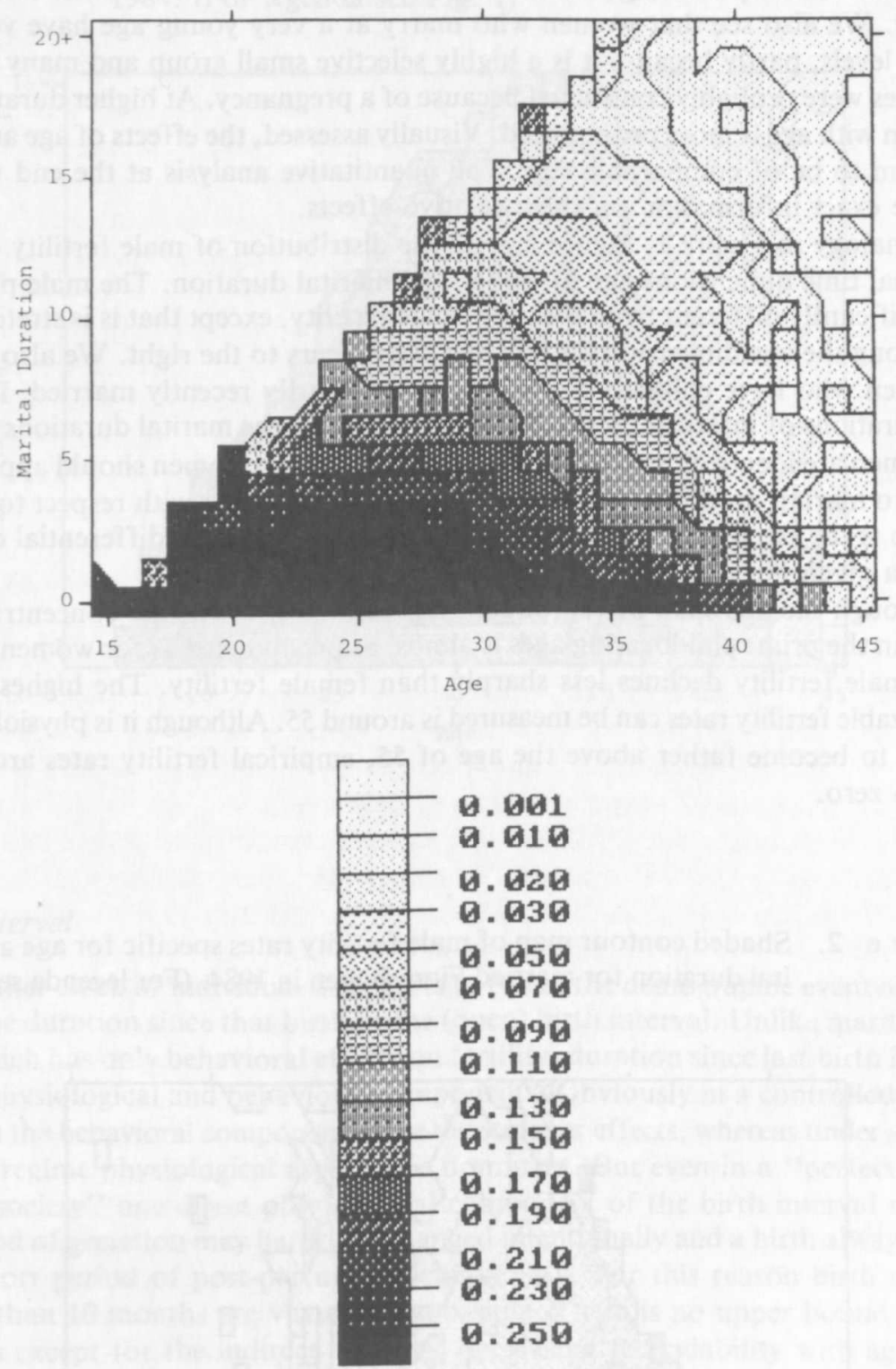

we also expect a decrease of fertility with increasing marital durations. Both expectations are clearly confirmed in Figure 1, but the patterns of decline with age and duration, appear to be quite different.

There are two parts of the figure where the rates are zero. The triangle in the upper left corner of the contour map is empty by definition because with a minimum age at marriage of 15 , certain combinations of age and duration are impossible. At the very right side of Figure 1 cells are not empty but fertility is empirically close to zero in most cases because few women above age 40 have children. We can see 
that at almost every age fertility is highest at duration zero especially up to age 35 . This high incidence of fertility shortly after marriage is not surprising when we consider that a sizable percentage of marriages is contracted when a child is already under way. We also see that women who marry at a very young age have very high fertility levels, partly because it is a highly selective small group and many of these marriages were probably contracted because of a pregnancy. At higher durations the variation with age is more pronounced. Visually assessed, the effects of age and duration seem to be of comparable size. The quantitative analysis at the end will give us more exact information on these relative effects.

In analogy to Figure 1, Figure 2 gives the distribution of male fertility over the individual time dimensions age of father and marital duration. The male pattern is not significantly different from that of female fertility, except that is is stretched out more along the age dimension and shifted a few years to the right. We also see that those men who have children above age 40 are mostly recently married, i.e. have short durations of marriage. Since for married couples the marital durations for men and women are identical, no difference between men and women should appear with respect to marital duration. Hence the marginal distribution with respect to marital duration is the same and the interest of our analysis lies in the differential distribution of a given duration-specific fertility over age.

Although the age span of reproduction is longer for men, the concentration of fertility in the prime childbearing ages is almost as pronounced as for women. Above age 40 male fertility declines less sharply than female fertility. The highest age at which sizable fertility rates can be measured is around 55. Although it is physiologically possible to become father above the age of 55 , empirical fertility rates are almost equal to zero.

F i g u r e 2. Shaded contour map of male fertility rates specific for age and marital duration for married Finnish men in 1984. (For legenda see Fig. 1)

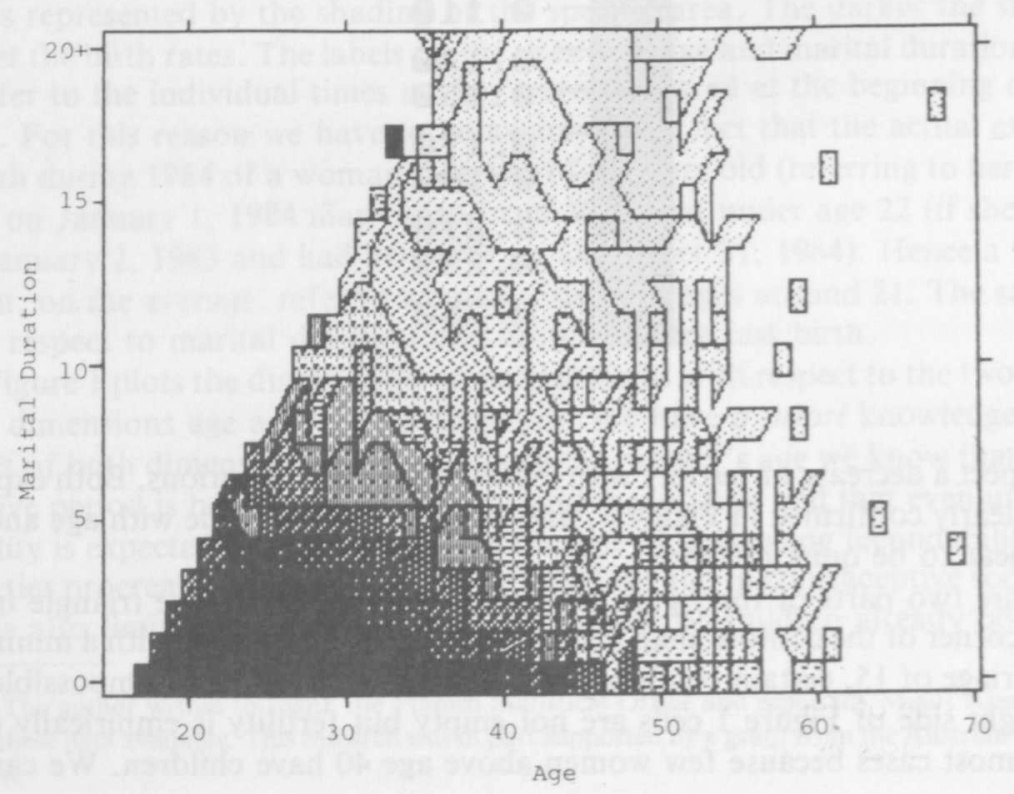


F i g u r e 3. Shaded contour map of fertility rates specific for age and duration since last birth for all Finnish women with parity one or higher in 1984. (For legenda see Fig. 1)

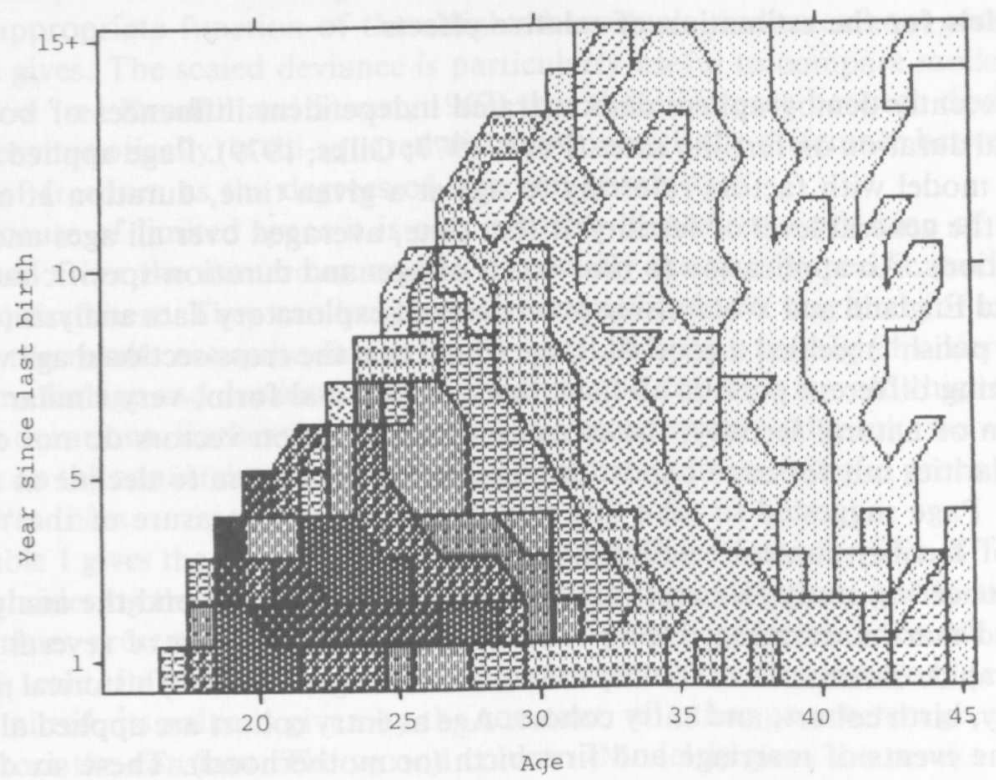

\section{Birth interval}

Another clock of individual time starts to run at the demographic event of a birth itself: the duration since that birth or the (open) birth interval. Unlike marital duration, which has only behavioral effects on fertility, duration since last birth has both strong physiological and behavioral components. Obviously in a controlled fertility situation the behavioral components have the stronger effects, whereas under a natural fertility regime physiological aspects are dominant. But even in a "perfect contraceptive society" one direct physiological component of the birth interval remains: the period of gestation may hardly be changed intentionally and a birth always causes some short period of post-partum infecundability. For this reason birth intervals shorter than 10 months are virtually impossible. There is no upper bound to birth intervals except for the indirect effect of decreasing fecundability with age.

Figure 3 gives the distribution of fertility over the two-dimensional plane defined by age and time since last birth. The analysis was restricted to all women (also unmarried) that had had at least one birth. From the contour map we see that fertility is highly concentrated within a relatively short span of birth intervals. The peak fertility rates clearly appear for birth intervals between two and four years ${ }^{2}$ and within the age span of 20 to 30 . From this peak fertility declines along both dimensions.

2 As with age and marital duration, a birth interval measured as, for example 2, at the beginning of 1984, could indicate an actual birth interval of between 2.0 to almost 4.0 years by the time a child is born in 1984. Because of a coding problem in the data set, we do not have unambiguous information on birth interval zero, which was left out for this reason. 
The decline toward the upper right corner is more symmetric than in the case of age and marital duration (see Figure 1).

\section{Logit models for the estimation of relative effects}

Only recently demographers demonstrated independent influences of both age and marital duration on fertility rates (Page, 1977; Gilks, 1979). Page applied a multiplicative model with factors referring to age at a given time, duration at a given time, and the general level of fertility at that time, averaged over all ages and marriage durations. An application to time-series of age- and duration-specific rates for Sweden and England and Wales using procedures of exploratory data analysis ("trimmed mean polish"') yielded a good fit. Page found that the cross-sectional age vectors characterizing different periods all have the same general form, very similar to the age pattern of natural fertility; the cross-sectional duration vectors do not exhibit clear regularities but for each cohort the duration effects seem to decline at a constant rate. Page suggested to take this rate of decline as a measure of the "latent intensity of fertility control"' within a cohort.

In a pioneering study Hobcraft and Casterline (1983) go beyond the analysis of the age and marital duration. Using World Fertility Survey data of several LDCs six demographic dimensions of fertility are considered: age, duration, historical period, age at entry, birth cohort, and entry cohort. Age at entry cohort are applied alternatively to the events of marriage and first birth (or motherhood). These six dimensions as specified by Hobcraft and Casterline, however, are not independent. In this way, for example, marital duration is equal to age minus age-at-marriage. Using GLIM (General Linear Interactive Modelling) with logarithmic link functions, Hobcraft and Casterline estimate several models with different numbers of demographic dimensions as explanatory variables. The main results for the nine LDCs studied are: age and duration since entry (to marriage or motherhood) are the dominant demographic dimensions; the patterns of age and duration effects prove to be very similar across countries. The age patterns are more heterogeneous than the duration patterns. Hobcraft and Casterline conclude from their models that the age pattern is more reflective of the level of intentional fertility control than the duration pattern. The two other important demographic dimensions of fertility, parity and birth interval for higher order births, were not considered in their study.

From these two studies we can already see that the approach to the consideration of simultaneous effects of several demographic dimensions is highly dependent on the structure of available data. While Page used time-series data from official vital registration and Hobcraft and Casterline used retrospective data from sample surveys, our model here will be based on the Finnish population register observing the events over one calendar year. This setting is not appropriate for cohort analysis, and past events such as the age at marriage could only be inferred indirectly. For this reason we will take a clear period approach considering independent demographic dimensions as they are measured at the beginning of 1984. The three continuous demographic dimensions of fertility that will be considered here are age, marital duration, and duration since last birth. The models were calculated for all women and men and also separately for women of specific parities.

The models were all fitted using GLIM (General Linear Interactive Modelling, release 3.77 ) with a logistic link function (and dummy variables for each age, duration, and birth interval), a binomial error structure taking the exposure as an offset and using a maximum-likelihood approach known as interative weighted least squares. 
Because the deviations of the data points from those predicted by the model must be weighted according to both the size of the denominators and the values fitted by the model, several cycles are required using the old estimates to obtain better ones. After the process has converged to a given degree of accuracy, the deviance, which is an appropriate function of the weighted residuals, indicates how good a fit the model gives. The scaled deviance is particularly useful to compare models because we know (see Kendall and Stuart, 1967) that the difference between deviances follows (asymptotically) a chi-squared distribution with the difference between the degrees of freedom as the degrees-of-freedom parameter.

Because of limited space it is only possible to present a selection of the results obtained from the numerous models. Especially the parity-specific models cannot be discussed here. They can be generally summarized in a way that for lower order births the results are quite similar to the total, whereas for higher order births the patterns for age and duration shift in the expected direction towards higher ages and longer durations. Rather than presenting too many numerical results we will put emphasis on the conversion of the resulting parameters to meaningful probabilities and interpret them.

Table 1 gives the resulting parameter estimates for women for four different models, considering the lone and combined effects of age, duration, and birth intervals. The values presented result from the estimation procedure and are still on the log (odds) scale. In each model the dummy variable of the first category (e.g. age 15) was omitted, its value is given by the constant; all other parameters give the deviation from the constant. The second column (Model I) gives the results from three completely independent runs where each dimension was taken separately as the sole explanatory variable of fertility rates. In case of age and time since last birth the estimates are given for all women and currently married women separately. Column 3 (Model II) gives the result of a model where age and duration were considered simultaneously. In Columns 4 and 5 the combinations of age plus birth interval and duration plus birth interval are given. ${ }^{3}$ In columns 3 to 5 the constant always stands for the combination of the two lowest categories, e.g. age 15 at duration zero in column 3. For models including birth intervals only women with at least one child were selected; for models including marital duration the analysis was restricted to married women. For all other cases all Finnish women between ages 15 and 49 were included.

Although some patterns can already be detected from the table, most of the interpretation needs a combination and transformation of parameters. To calculate the probability of birth e.g. for women aged 20 at marital duration three (Model II) one takes the constant and adds the parameters of age 20 and duration three to it. This is still the logarithm of the odds ratio, and further algebraic transformation is needed to get the probability, which is .235 in the above example. The following figures will present a selected series of such probabilities for one dimension when controlling for another.

\section{Interpretation of the logit coefficients}

Figure 4 plots the age-specific birth probabilities for married women in Finland in 1984, controlling for marital duration, based on results of Model I. We clearly see that generally, lower marital duration means higher birth probabilities at all ages.

${ }^{3}$ A simultaneous consideration of all three dimensions was not conducted because it would be excessive in terms of computer space and time, and the results would be extremely difficult to interpret. 
T a b l e 1. Relative effects of age, marital duration, and time since last birth on the fertility of Finnish women in 1984 (coefficients from a logit model).

(1)

Age

Scaled deviance

Constant

16

17

18

19

20

21

22

23

24

25

26

27

28

29

30

31

32

33

34

35

36

37

38

39

40

41

42

43

44

45

46

47

48

49

50

Marital

duration

Scaled deviance

Constant

$\begin{array}{cc}1 & -0.4301 \\ 2 & -0.5942 \\ 3 & -0.7086 \\ 4 & -0.8117 \\ 5 & -1.020 \\ 6 & -1.221 \\ 7 & -1.468 \\ 8 & -1.638 \\ 9 & -1.864 \\ & -2.019\end{array}$

Model I separately

Married

$$
8439
$$

(3)

(4)

(5)

Each effect

considered

All Married

$9750 \quad 13847$

$-0.6931 \quad 7.226$

$\begin{array}{ll}-0.4855 & -6.938\end{array}$

$-0.9014 \quad-7.462$

$\begin{array}{ll}-0.5315 & -7.544\end{array}$

$\begin{array}{ll}-0.5699 & -7.585\end{array}$

$-0.6323 \quad-7.902$

$\begin{array}{ll}-0.6570 & -8.020\end{array}$

$-0.7190 \quad-8.184$

$\begin{array}{ll}-0.8009 & -8.244\end{array}$

$\begin{array}{ll}-0.8093 & -8.283\end{array}$

$\begin{array}{ll}-0.8736 & -8.324\end{array}$

$\begin{array}{ll}-0.9854 & -8.470\end{array}$

$\begin{array}{ll}-1.060 & -8.568\end{array}$

$-1.147 \quad-8.682$

$\begin{array}{ll}-1.269 & -8.829\end{array}$

$-1.351 \quad-8.966$

$-1.569 \quad-9.201$

$-1.703 \quad-9.347$

$-1.869 \quad-9.548$

$-2.050 \quad-9.748$

$\begin{array}{ll}-2.272 & -9.954\end{array}$

$-2.443-10.12$

$-2.664-10.36$

$-2.941-10.65$

$\begin{array}{ll}-3.320 & -10.98\end{array}$

$-3.603-11.24$

$\begin{array}{ll}-4.041 & -11.59\end{array}$

$\begin{array}{ll}-4.574 & -12.09\end{array}$

$\begin{array}{ll}-5.326 & -12.61\end{array}$

$\begin{array}{ll}-5.866 & -13.08\end{array}$

$\begin{array}{ll}-7.267 & -13.72\end{array}$

$\begin{array}{ll}-7.806 & -14.36\end{array}$

$-9.334-15.50$

$\begin{array}{ll}-12.31 & -20.04\end{array}$

$-11.90 \quad-19.92$

$-11.79 \quad-19.73$

$-0.5971$

$-0.6881$

$-0.7795$

$-0.9692$

$-1.156$

$-1.383$

$-1.530$

$-1.715$

$-1.819$
Model III

Combined

effects of age

and birth interval

All
2200

$-0.6931$

$-0.2393$

$-0.4618$

$-0.1120$

$-0.06310$

$-0.09005$

$-0.07245$

$-0.1010$

$-0.1442$

$-0.1290$

$-0.1676$

$-0.2490$

$-0.2917$

$-0.3384$

$-0.4203$

$-0.4597$

$-0.6208$

$-0.6989$

$-0.8030$

$-0.9208$

$-1.072$

$-1.166$

$-1.315$

$-1.506$

$-1.796$

$-1.998$

$-2.364$

$-2.805$

$-3.487$

$-3.937$

$-5.277$

$-5.749$

$-7.229$

$-12.03$

$-11.61$

$-11.43$
Model IV

Combined effect of duration and birth interval

Married 3630

$$
\begin{aligned}
& -0.08762 \\
& -0.7084 \\
& -0.5188 \\
& -0.6101 \\
& -0.7825 \\
& -0.9454 \\
& -1.189 \\
& -1.336 \\
& -1.523 \\
& -1.630
\end{aligned}
$$


(1)

Age
(2)

Model I

Each effect

sor sidered

sep riately

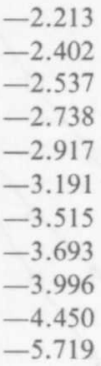

(3)

Model II

Combined

effects of age

and duration

$-1.958$

$-2.082$

$-2.149$

$-2.266$

$-2.354$

$-2.513$

$-2.707$

$-2.736$

$-2.854$

$-3.077$

$-3.095$
(4)

(5)

Model IV

Combined

effect of

duration and

birth interval

$-1.781$

$-1.927$

$-1.996$

$-2.132$

$-2.237$

$-2.429$

$-2.624$

$-2.703$

$-2.882$

$-3.179$

$-4.009$

Years since last

birth (only

women with

parity 1 or

more)

Scaled deviance

$\begin{array}{cc}\text { All } & \text { Married } \\ 13124 & 16496 \\ -1.156 & -1.041 \\ -0.9619 & -0.9124 \\ -0.6928 & -0.6152 \\ -0.9495 & -0.8650 \\ -1.206 & -1.127 \\ -1.543 & -1.483 \\ -1.718 & -1.674 \\ -1.903 & -1.857 \\ -2.147 & -2.097 \\ -2.414 & -2.388 \\ -2.564 & -2.495 \\ -2.833 & -2.796 \\ -3.006 & -3.002 \\ -3.266 & -3.257 \\ -3.644 & -3.646\end{array}$

-0.9459
-0.5961
-0.7661
-0.9271
-1.168
-1.255
-1.345
-1.484
-1.639
-1.671
-1.809
-1.840
-1.938
-2.135

$-0.8727$

$-0.4379$

$-0.5062$

$-0.5834$

$-0.7685$

$-0.8103$

$-0.8689$

$-1.002$

$-1.179$

$-1.182$

$-1.362$

$-1.454$

$-1.580$

$-1.845$

The decline seems to be steepest between durations zero and one. In Figure 5 we will see that for durations one to four the decline is also somewhat slower than for the high durations up to about 8 years. Below age 20 the pattern is irregular because the number of women married at that age is very small and selective. To a demographer the most exciting finding from this graph is that the curve of age-specific fertility at low marital durations and especially at duration zero is concave to the origin such as that in a completely natural fertility population without fertility control. And, indeed, it is very plausible that even in a highly contraceptive modern society fertility within the first years of marriage is practically uncontrolled even for women above age 35 . Because marriage and childbearing are still so closely related even in an industrialized society with low fertility, newly married women in their late thirties have significantly higher fertility than other married women at the same age.

To illustrate the difference between the general pattern of age-specific marital fertility and that for women with short marital durations, Figure 4 also plots the age pattern for women of all marital durations. We see that the decrease with age is initially steeper for all married women and the line actually crosses the curves that con- 
F i g u r e 4. Estimated probabilities of age-specific female fertility for selected marital durations.

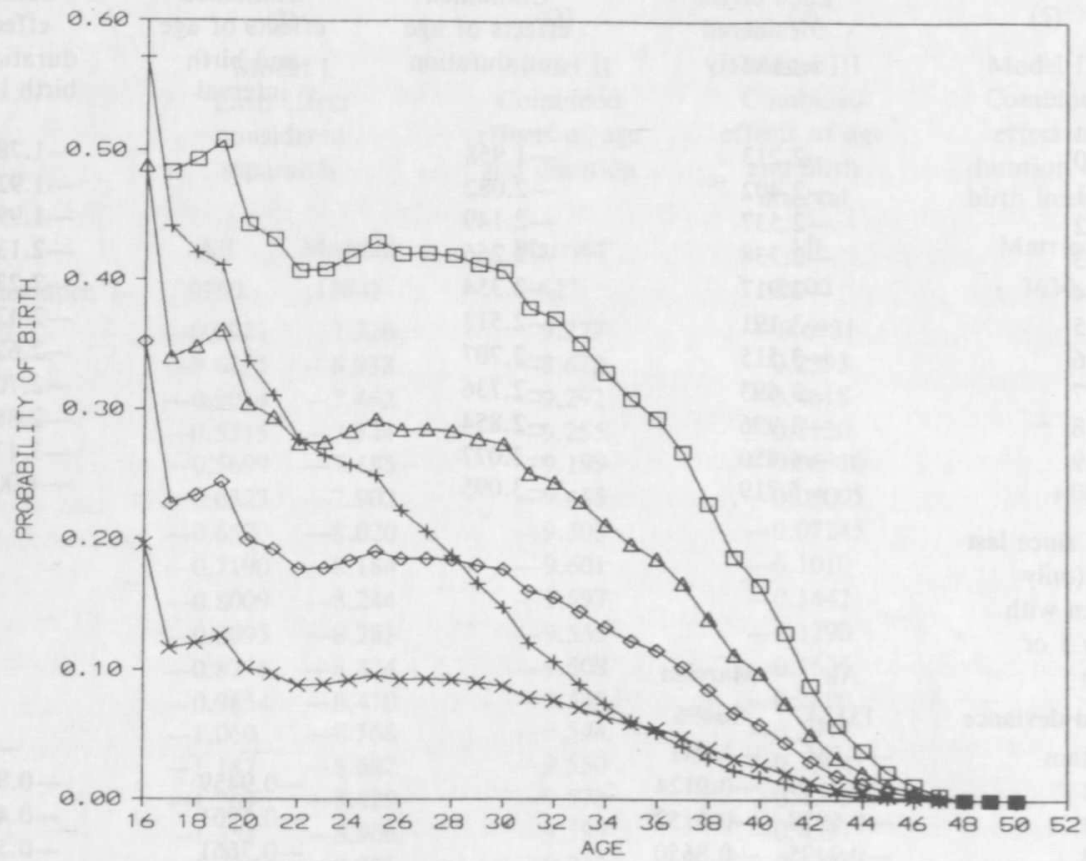

DDURATION $\oslash$ DDUR 1 ODUR 5 XDUR 10 + ALL DURS

F i g u r e 5. Estimated probabilities of marital duration-specific female fertility for selected age groups.

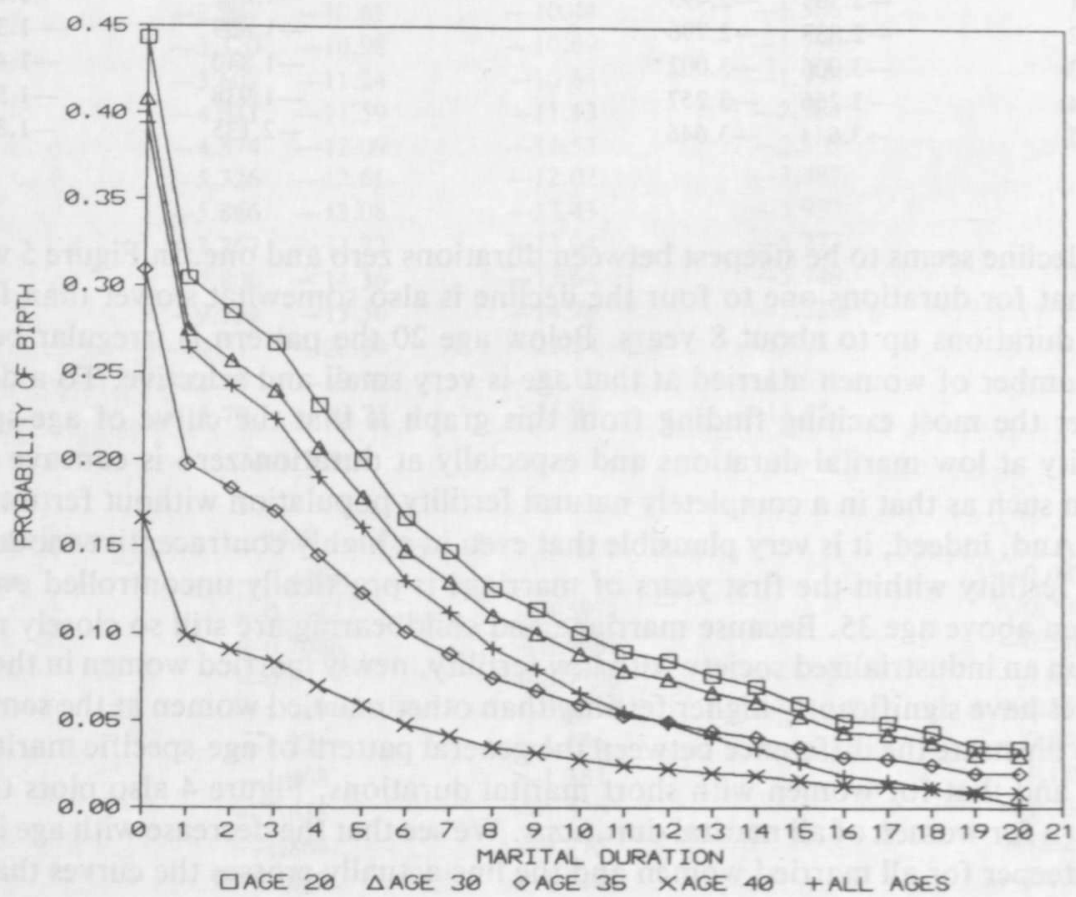


F i g u r e 6. Estimated probabilities of female fertility specific for time since last birth for selected age groups.

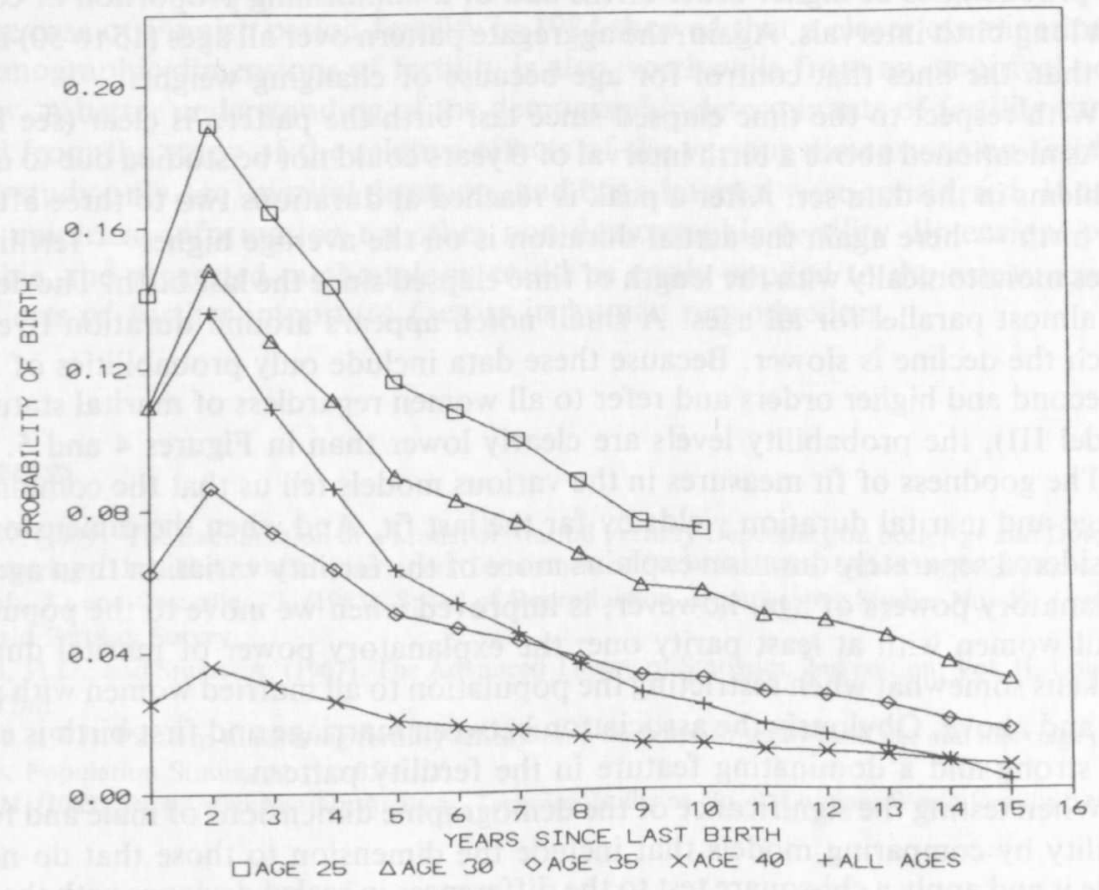

trol for duration. The statistical reason for these cross-overs lies in the change of weights with age: in the lower twenties women with short marital duration and therefore high fertility dominate the picture, whereas women that are newly wed are rare in the late thirties and their relatively high fertility has little impact on the total level. From this we can already conclude that marital duration has a very significant impact on fertility. If the lines were horizontal then the picture would be completely dominated by duration and an appearing age effect in the aggregate distribution including all durations would be an artefact only caused by the change in the marital duration composition of the population. As we see, this is almost the case between ages 20 and 30 . After that, however, fertility declines with age, even at fixed marital durations.

Figure 5 turns the perspective around and looks at the fertility patterns along the dimension marital duration when controlling for age. We find a distinct pattern that seems to hold across all ages: fertility is by far the highest at duration zero-here again duration zero actually means a duration which is on the average .5 to 1.5 yearsdeclines sharply to age 1 , and then shows a monotonic decline which is steepest between durations three and eight. This pattern is more pronounced for younger ages. It is not difficult to speculate about the reasons of this pattern. In Finland - as in many other European countries - often marriages are contracted because the woman is already pregnant and probably many conceptions take place shortly after marriage. Hence the high level at duration zero and the decline to duration one is plausible; the slower decline in birth intensities between durations one and four is probably due to the second round of children coinciding with the fertility of couples that delayed their first births for a few years. From the following analysis of birth 
intervals we also see that 2 to 3 years is a very common interval. Generally the monotonous decline of birth probabilities with marital duration is a consequence of the low probabilities of higher order births and of a diminishing proportion of couples with long birth intervals. Again, the aggregate pattern over all ages (15 to 50) is steeper than the lines that control for age because of changing weights.

With respect to the time elapsed since last birth the pattern is clear (see Figure 6). As mentioned above a birth interval of 0 years could not be studied due to coding problems in the data set. After a peak is reached at durations two to three after the last birth - here again the actual duration is on the average higher - fertility declines monotonically with the length of time elapsed since the last birth. The declines are almost parallel for all ages. A small notch appears around duration five after which the decline is slower. Because these data include only probabilities of births of second and higher orders and refer to all women regardless of marital status (see Model III), the probability levels are clearly lower than in Figures 4 and 5.

The goodness of fit measures in the various models tell us that the combination of age and marital duration yields by far the last fit. And when the dimensions are considered separately duration explains more of the fertility variation than age. The explanatory powers of age, however, is improved when we move to the population of all women with at least parity one; the explanatory power of marital duration weakens somewhat when restricting the population to all married women with parity one and above. Obviously the association between marriage and first birth is especially strong and a dominating feature in the fertility pattern.

When testing the significance of the demographic dimensions of male and female fertility by comparing models that include the dimension to those that do not include it and apply a chi-square test to the differences in scaled deviance with the given difference in degrees of freedom, the results may be summarized in the following way: for every model presented here the reduction in deviance due to the inclusion of a second dimension into a model that already included another dimension was highly significant (a probability level of .995). In other words, the Finnish data give extremely strong evidence that there is a real effect of marital duration on fertility in addition to age, of birth interval in addition to age, of duration in addition to birth interval, of age in addition to marital duration, etc. Since this test only concerns the complete dimension variable with all ages, durations, or intervals, it does not give us information about the relative importance of the dimensions within restricted age spans, duration spans, etc. There is no theoretical reason not to conduct such tests, although in practice it would require innumerable models to be fitted, a task that goes beyond our objective here. On the basis of the presentation of estimated patterns of probabilities (see Figures 4 to 6), however, one can make inferences on the change of relative importance at various ages or durations. One such example is fertility at duration 0 (see Figure 4) which between ages 22 and 30 remains virtually constant with respect to age, i.e shows no effect of age.

\section{Outlook}

To speak of demographic dimensions is still uncommon among demographers. Very few authors (e.g. Hobcraft and Casterline, 1983) have been explicitly referring to this notion. We believe that the rather general concept of seeing a demographic event registered in a space put up by various demographic and non-demographic dimensions is very helpful in understanding the basic philosophy of the approach of measurement one uses and to understand it as one approach out of many possible 
ones. It seems to be a concept that is also wide enough to cover essentially all of the demographic approaches taken so far and could help to provide conceptual clarity in more difficult questions such as the age-period-cohort question.

The case of Finnish period fertility in 1984 showed that a closer examination of the demographic dimensions of fertility is also worthwhile from an empirical point of view. A better understanding of the demographic determinants of fertility can be gained from the study of the relative effects of the various dimensions on fertility. In this study only age, marital duration, and birth interval were considered. If additional empirical information on other non-demographic fertility dimensions were available, the suggested methodology could be easily applied to the assessment of the effects of further important factors in human reproduction.

\section{References}

Gilks, W. (1979). The Examination of a Model of Marital Fertility Depending on both Age and Duration of Marriage. Unpublished MSc dissertation, Department of Social Statistics, University of Southampton.

Hobcraft, J.; and Casterline, J. (1983). Speed of Reproduction. Comparative Studies No. 25. London: World Fertility Survey.

Kendall, M.G.; and Stuart, A. (1967). The Advanced Theory of Statistics, 2nd edition, Vol. II. London: Griffin.

Page, H. (1977). Patterns underlying fertility schedules: a decomposition by both age and marriage duration. Population Studies 31 (1): 85-106.

Ryder, N. (1982). Progressive Fertility Analysis. Technical Bulletins No. 8. London: World Fertility Survey. 